\title{
The Morphology and Motility of Proteus vulgaris and Other Organisms Cultured in the Presence of Penicillin
}

\author{
By A. FLEMING, AMALIA VOUREKA, I. R. H. KRAMER \\ AND W. H. HUGHES \\ The Wright-Fleming Institute of Microbiology, London, W.2
}

\begin{abstract}
SUMMARY: Microbes were grown on microscope slides so that the growth could readily be observed by phase-contrast microscopy.

Proteus vulgaris, grown on agar containing penicillin, undergoes extraordinary morphological changes which vary with the temperature of incubation, the concentration of the penicillin, the concentration of the agar and the presence of small amounts of fluid between the agar and the cover-slip. The bacilli may divide normally once or twice into elements that grow without dividing and which may develop into fantastically shaped thread or swollen forms. In high concentrations of penicillin the fantastic shapes are obtained by enlargement without division. At first the nuclei divide as in normal organisms. The thread forms have condensed nuclei arranged in alternating pattern along the side of the cells. In the swellings there may be either nuclear material filling the cells, a condensed central mass or a reticulum. When vacuoles are present these displace the nuclear material.
\end{abstract}

When the misshapen organisms are transferred to a medium free from penicillin and containing penicillinase they divide, forming normal bacilli. Many of the swollen elements burst and disappear.

The motility of the greatly enlarged organisms is sluggish, and flagellar movement can clearly be observed by phase contrast. The movement of the flagella of the organisms responds readily to radiant heat, and a careful study of these movements makes it impossible to accept Pijper's contention that bacterial motility is due entirely to undulatory movements of the body and that the flagella are merely mucoid strands cast off as the result of motility.

The flagella were demonstrated in the large forms by fixing the culture through the agar for several days, detaching the agar and staining the cover-slip, which carries the fixed colony, with a saturated watery solution of night blue. The nuclei were shown by treating films with hot nitric acid, washing and staining first with cresyl blue then Leishman's stain.

It was pointed out by Gardner (1940) that bacteria grown in non-lethal concentrations of penicillin exhibit a grossly modified morphology resulting apparently from a failure of cell division. Among the cocci swollen and bloated forms appear and the bacilli form long threads. Gardner's observations have been confirmed by many observers. Similar changes, but perhaps not so extensive, have been noted after exposure of bacteria to other chemicals (Ainley Walker \& Murray, 1904; Spray \& Lodge, 1943; Webb, 1948; for a review of the literature see Klieneberger-Nobel, 1949).

None of the workers, however, has illustrated clearly the completeness of the morphological changes which can be induced by penicillin on relatively insensitive organisms like Proteus vulgaris.

$P$. vulgaris is an easy organism to work with; it grows rapidly at room 
temperature and with penicillin grows in the most fantastic forms. Moreover, it is a highly motile organism possessing many flagella and serves well for a study of the functions of these flagella.

\section{MATERIALS AND METHODS}

Microscopy. Phase-contrast microscopy has enabled us to observe clearly the growth of $\boldsymbol{P}$. vulgaris on agar containing penicillin in various concentrations. Much more dramatic results were obtained with cultures on agar than with cultures in a fluid medium, and there was a great difference between cultures grown at $37^{\circ}$ and at room temperature $\left(18-20^{\circ}\right)$. At the lower temperature the changes took place more slowly: there was more bacterial growth and in many ways the morphological changes were clearer. The cultures at room temperature were much more valuable for studying motility and flagellar action.

For the most part cultures were grown on microscope slides and were examined by phase contrast. Under a $\frac{1}{6}$ th dry objective the culture could be examined repeatedly or continuously without even the slight disturbances which might result if an oil immersion lens was used. For the observation of flagella, however, a $\frac{1}{12}$ in. oil immersion was necessary.

Slide-culture technique. Slide cultures for continuous examination were made in two ways:

(1) Penicillin agar was spread in a thin layer on a sterilized microscope slide. The slide was heated and with a pipette the melted agar distributed as evenly as possible over the central portion of the slide. When the agar had set it was trimmed to a square with the edge of another slide so that it was a little smaller than the cover-slip to be used. A small loopful of a dilution of a young $\boldsymbol{P}$. vulgaris culture was placed on the agar, and a sterile cover-slip applied. Small irregularities on the surface could, if desired, be made before the agar was inoculated, so that it was possible to observe the bacilli: $(a)$ with the cover-slip in direct contace with the agar; $(b)$ with a very thin layer of fluid between the cover-slip and the agar in which the bacilli had some free movement; and (c) a deeper pool of fluid in which the movements were quite unhindered.

On removing the cover-slip from such a culture there was an enormous disturbance of the bacteria so that it was unsuitable for the preparation of permanent stained specimens. When permanent specimens of the undisturbed culture were required, or when we wished the whole of the agar to be in contact with the cover-slip, the preparations were made as follows:

(2) The culture is spread on a sterile cover-slip and allowed to dry. Then penicillin agar (1-1.5\% agar) at a temperature under $50^{\circ}$ is dropped on the cover-slip and as soon as it has set the cover-slip is inverted on a slide. When the cover-slip is picked up with a pair of forceps the whole of the agar adheres to it and there is almost no disturbance of the culture. The cover-slip with the agar can be fixed in formalin and when fixation is complete the agar can be gently removed leaving on the cover-slip an almost undisturbed pattern of the 
bacilli in the culture which can be stained to give a beautiful permanent record of the culture.

In addition to simple staining the bacilli were treated to show their nuclei and their flagella.

Flagella staining. Slide cultures made by method (2) were used. They were observed by phase contrast and if flagella were seen the cover-slip with the agar attached was picked off and placed in formalin in a Petri dish. After 2 days' or more fixation the agar was gently removed from the cover-slip. This was washed to remove all traces of the culture medium and was then stained. Kirkpatrick's staining method gave fairly good results and when this was followed by staining with carbol fuchsin for $5 \mathrm{~min}$. the flagella were much more prominent.

Later we found that simple watery solutions of certain dyes stained the flagella well after prolonged formalin fixation and the best we found to be a saturated watery solution of night blue applied for $5 \mathrm{~min}$. at room temperature.

Nuclear staining. The demonstration of nuclear material was long impeded by the staining affinity of the cytoplasm for basic dyes after fixation. Various methods have overcome this disadvantage (see Robinow, 1945).

Another acid hydrolysis staining technique was devised by one of us (I. R. H. K.) and used to study the distribution of nuclear material in our cultures.

Film or impression preparations are air-dried. A Petri dish is filled with a $7 \% \mathrm{v} / \mathrm{v}$ dilution of concentrated nitric acid in distilled water. This is heated to boiling point and allowed to cool. When cold, the slide is placed in the solution, which is again heated until 'bumping' starts. The slide is at once removed from the acid and washed for 15-30 sec. in running tap water. Excess water is shaken off and the slide flooded with $1 \%$ brilliant cresyl blue in $0.9 \%$ saline. After 15-30 sec. this solution is washed off with tap water, the slide rinsed with distilled water, and flooded with Leishman's stain which is then diluted with an equal volume of distilled water. Staining is usually complete in 30 sec. or less, when the slide is washed with distilled water and blotted dry or mounted wet. The nuclear material is stained blue-black and the rest of the cell pink. The intensity of the counter-staining is increased by prolonging the washing in distilled water before the Leishman solution is applied. It has been found that with some batches of brilliant cresyl blue, more selective staining is obtained if the solution has been kept for a few months.

\section{RESULTS}

The effect of penicillin on growth and morphology

High concentrations of penicillin in agar. The strain of $\boldsymbol{P}$. vulgaris used in these experiments was, after $24 \mathrm{hr}$., completely inhibited by 12.5 units (u.) of penicillin/ml.

Experiments were made with concentrations in the agar of from 1 to $3000 \mathrm{u} . / \mathrm{ml}$. The results varied greatly with the concentration but even with $3000 \mathrm{u} . / \mathrm{ml}$. there was evidently some growth, although there was no cell 
division. Some organisms enlarged to 50 or 100 times their normal size, became rounded and swollen and resembled some strange protozoon rather than a bacterium.

The most common forms obtained with different concentrations of penicillin are illustrated in Fig. 1.

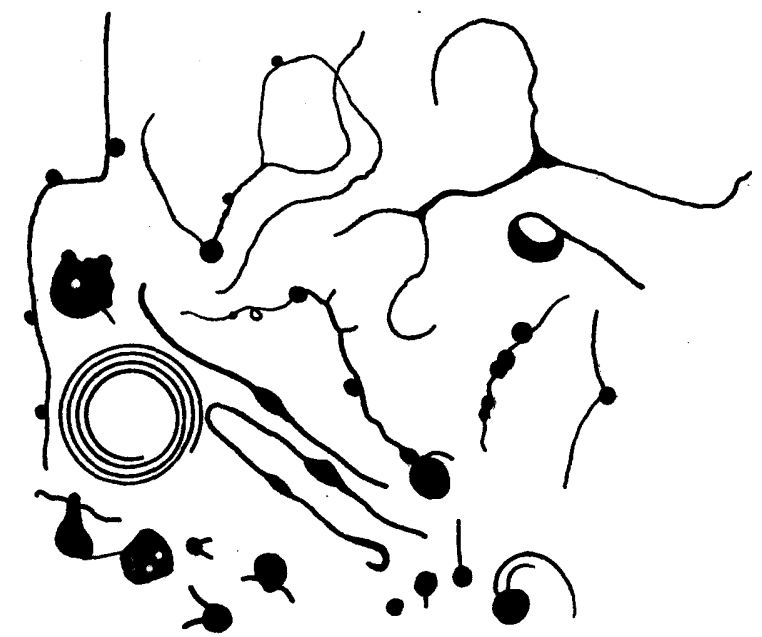

Fig. 1

Low concentrations of penicillin in agar. According to the physical conditions (strength of agar, presence of fluid, etc.) there were great variations in the morphology of the organisms. PI. 1, figs. 1-6, which are photographs of the same bacteria during a period of $19 \mathrm{hr}$. at room temperature, gives a fairly typical picture of the development of a bacillus where the agar and the coverslip were in apposition. The organism, after a period of $1-1 \frac{1}{2} \mathrm{hr}$., divides and the daughter cells appear normal. These may again divide producing normal or somewhat larger cells. After one or two normal divisions, however, the cells elongate without division and this elongation may be very great. Very frequently these long forms coil themselves into watch-spring-like spirals and rotate slowly or rapidly without changing their position in the microscopic field (Pl. 1, figs. 4, 5; Pl. 3, fig. 28).

Meanwhile, bulbous enlargements develop in many of the rods (Pl. 1, fig. 5). There may be one or many along the length of the filament, lying centrally in the axis of the filament or forming excrescences on one side or other. Often when a spiral form develops a large bulb, it rotates with this bulb as its axis as though the bulb had made a depression in the agar so that the organism was anchored except for a rotary movement with the bulb at the centre. These bulbs remain small, or enlarge until they are 20 or more times the diameter of the filament, which itself is 2 or 3 times the diameter of the normal bacillus.

There is a great variation in the individual organisms. Some grow into filaments with or without bulbous enlargements; some develop bulbs without much elongation and some are perfect spheres which may be actively motile. 
Where there is free fluid many of the organisms do not appear to change. It is common to see practically all the organisms misshapen where the agar is in contact with the cover-glass, but round the edges where there is free fluid there are myriads of actively motile and apparently normal bacilli mixed with a few abnormal forms.

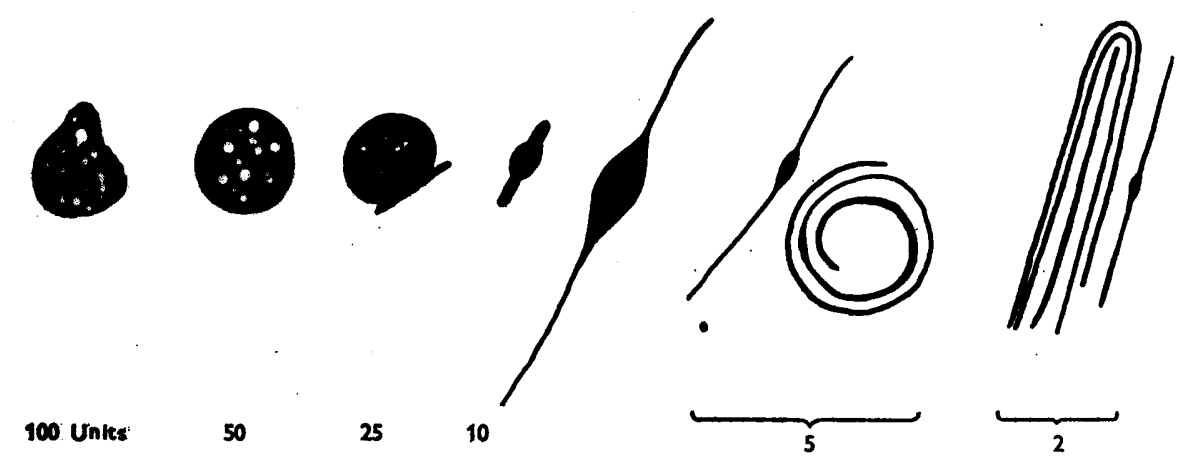

Fig. 2

With higher concentrations of penicillin, up to $25 \mathrm{u} . / \mathrm{ml}$., there is perhaps one normal division and then cells elongate into filaments with or without bulbs or develop bulbs with little or no filamentous expansion. When there is a very thin layer of fluid under the cover-slip it is not uncommon to find extraordinary branched forms (Pl. 3, fig. 26). These are sluggishly motile and often only one branch may be moving at one time.

With still higher concentrations of penicillin there is no division and many of the organisms remain unchanged; but many, on the other hand, develop into large bulbs perhaps with one or two short projections representing the end or ends of bacilli (Pl. 3, fig. 27). Fig. 2 illustrates some of the bizarre forms which may be seen in $24 \mathrm{hr}$. slide cultures at room temperature.

Imitation of bathing pool or sun-bathing beach. Apart from the activity of the individual organisms certain activities on the part of the group may be noticed. When a low concentration of penicillin (say $5 \mathrm{u} . / \mathrm{ml}$.) is used in the agar and there is a slight irregularity of the surface so that a shallow pool with a shelving edge is formed under the cover-glass, a very good imitation is seen of a sun-bathing beach. The shelving edge is covered with radially arranged long bacilli, just like people stretched out on the sands. Every now and then one swims off into the pool and its place is taken by another. This is best seen before the organisms have developed bulbs, otherwise the bulbous ones seem to become anchored and cannot leave the beach. Pl. 2, fig. 18, illustrates such a pool. Here many of the organisms actually swimming in the fluid are bulbous. They are too large to get back to shore.

\section{Penicillin-induced change in nuclear material}

There is a regular sequence of development of the abnormal forms, and though it does not take place at the same rate in all the bacilli the order of stages appears to be constant. The general morphology of the types has been 
described; the flagella are dealt with below; the nuclei at the various stages are as follows.

Stage 1. The small cells have nuclei in the positions described by Robinow (1945) for normal cells; but these lose their dumb-bell form and become rounded (Pl. 2, figs. 7-9).

Stage 2. Long filaments have nuclei similar in appearance to those of normal cells but repeated along the threads. All stages of division may be seen in one thread, and dumb-bell forms are more easily distinguished. In some threads, probably those more affected or exposed for longer periods, nuclear division ceases, the nuclei condense into dots which are often arranged along the thread on alternate sides, in contact with the cell membrane.

Stage 3. Threads with swellings. The nuclei of the threads are similar to those of organisms in stage 2. In the swellings several different nuclear appearances are found. The nuclear material may fill the space (Pl. 2, fig. 13), be collected into a central mass (fig. 14) or form a coarse reticulum (fig. 18).

Stage 4. Spheres. These have the same type of nuclei as stage 3 or swellings (Pl. 2, fig. 16). When vacuoles are present these displace nuclear material (Pl. 2, fig. 17).

\section{Recovery from exposure to penicillin}

Pl. 3, figs. 22-25, give the sequence of events when organisms from the culture on penicillin agar in the series (Pl. 1, figs. 1-6) were transferred to agar containing penicillinase. The cover-slip was lifted from the block of penicillinagar and with the adherent organism transferred to a block of penicillinase-agar, resealed and the second series of photographs made.

Pl. 3, fig. 22, shows organisms of various shapes. After transferring to the penicillinase-agar the fusiform organism develops back into normal organism, separation of the ends taking place first. Next the bulbs divide across to give two club-shaped cells which in subsequent divisions approximate more and more to the normal bacillary forms.

The division of the filament and the multiplication of the young organisms derived therefrom results in a collection of normal bacilli round the large swellings, which burst and degenerate. But for the continuous observation the appearance might have suggested that the small forms were liberated from the bursting of a cyst-like formation. Nevertheless, organisms derived from spheres may occur.

\section{Motility and flagella in penicillin-induced forms}

Traditionally flagella are regarded as the organs of motility in bacteria, but recently Pijper (1946) has maintained that motility is due to 'gyratory undulating' movements of the bacillary body and the flagella are 'slime layer' strands thrown off because of the active motion. When one observes the short active bodies of Salmonella typhi or Proteus vulgaris the movement is too rapid for any conclusion about the nature of motility to be drawn. The case is quite different, however, with the large filamentous and bloated forms of $\boldsymbol{P}$. vulgaris grown in slide culture on penicillin agar. The activity of the organisms depended to some extent on the density of the agar and whether there was fluid between 


\section{Penicillin-induced changes in $\mathbf{P}$. vulgaris}

the cover-slip and the agar. When the agar was very soft or when there was the thinnest layer of fluid under the cover-slip, then at some stage, all or most of the organisms were motile at room temperature. When the agar was stiff and there was no fluid a large proportion of them were non-motile, especially when grown at $37^{\circ}$.

In all cases the motility of these enlarged organisms was sluggish compared with normal $\boldsymbol{P}$. vulgaris in a fluid medium, and the flagella could be observed directly by phase-contrast microscopy. Individual flagella were probably not visible but in these gross forms, when the filaments were close together, the flagella formed themselves into wavy rope-like strands which could easily be seen (Pl. 3, fig. 28).

When there was a considerable space between the filaments a wavy motion could be seen in the fluid indicating flagellar activity, although the actual flagella could not be seen.

The effect of radiant heat on motility. The motility of these organisms is very sensitive to heat. Heat filters which hardly affect the intensity of the light from the microscope lamp, stop motility when placed across the beam of light. This effect is not evident in the very active younger cultures nor is it seen in all the motile bacteria of a culture. It is most marked in cultures $24 \mathrm{hr}$. or more old, grown at room temperature, in which the motility is more sluggish.

The fact that these organisms exhibited active movements in the slide cultures and yet remained in the same microscope field made it easy to follow the effect of heat on their movements. Without a heat filter they were rotating or moving to and fro in a limited space. When a heat filter was imposed movement stopped. When the filter was removed after a latent period of from a fraction of a second to about 5 sec., the flagella were seen in active motion. Often there was no appreciable interval between the beginning of flagellar motion and the motion of the whole organism. This is exactly what would be expected if there were no physical obstacle to the movement of the body. Sometimes there was a distinct lag between the commencement of flagellar activity and movement of the bacillary body. This might be anticipated if the body is closely confined between the cover-slip and the agar when the flagellar activity would have to overcome considerable force before the body could move.

Effect of roithdrawal of the heat stimulus. Observations were made on the motility of some of the long coiled filaments which only rotated in the microscope field. Movement ceased as soon as a heat filter was placed in the light beam: when stimulated by some exposure to the heat rays the organism moved actively, but it soon became more sluggish and eventually motion ceased. We selected certain organisms and measured the time occupied in making each single revolution after a heat filter had been interposed for various times (Table 1a).

This is probably the first time that a bacillus has been timed for laps in a long distance race, and several interesting points emerge from the figures in Tables $1 a$ and $1 b$. A rest of 75 sec. gives exactly the same result as a rest of 180 sec. From this it could be argued that in 75 sec. the organisms had regained their maximum of stored energy. When the period of rest is only a few seconds 
Table 1a. Motility of a coiled organism after shielding from radiant heat for various periods

\begin{tabular}{|c|c|c|c|c|}
\hline . & & Revolutions afte & renewed stimulus & \\
\hline Duration of rest & Num & ber at & Time of a single & Total \\
\hline $\begin{array}{c}\text { stimulation } \\
\text { (sec.) }\end{array}$ & $\begin{array}{l}\text { Maximum speed } \\
\quad(2-5 \text { sec. })\end{array}$ & $\begin{array}{c}\text { Slower speed } \\
\text { before stopping }\end{array}$ & $\begin{array}{l}\text { the slower } \\
\text { speed (sec.) }\end{array}$ & $\begin{array}{c}\text { before } \\
\text { stopping }\end{array}$ \\
\hline 180 & 23 & 0 & - & 23 \\
\hline 2 & 7 & $\mathbf{1}$ & 13 & 8 \\
\hline 75 & 22 & 1 & $\mathbf{9}$ & 23 \\
\hline 10 & 13 & $\mathbf{1}$ & 8 & 14 \\
\hline 2 & 6 & 2 & 7,12 & 8 \\
\hline $\mathbf{5}$ & 9 & 2 & 8,25 & 11 \\
\hline 75 & 28 & 1 & 11 & 24 \\
\hline $\mathbf{1}$ & 2 & 2 & 12,26 & 4 \\
\hline 5 & 11 & 2 & 6,7 & 13 \\
\hline $\mathbf{2}$ & 7 & $\mathbf{0}$ & - & 7 \\
\hline 1 & $\mathbf{3}$ & 1 & 13 & 4 \\
\hline 180 & 24 & $\mathbf{0}$ & - & 24 \\
\hline
\end{tabular}

Table $1 b$. The relation of the duration of rest from heat stimulation, and subsequent revolutions of motile bacteria. Data from Table $1 a$, cols. 1 and 5

$\begin{array}{cccc}\begin{array}{c}\text { Duration of rest } \\ \text { from heat } \\ \text { stimulation }\end{array} & \begin{array}{c}\text { No. of } \\ \text { (sec.) }\end{array} & \begin{array}{c}\text { No. of revolutions } \\ \text { before motility } \\ \text { ceases }\end{array} & \text { Average } \\ 180 & 2 & 23,24 & 23 \cdot 5 \\ 75 & 2 & 23,24 & 23 \cdot 5 \\ 10 & 1 & 14 & 14 \\ 5 & 2 & 11,13 & 12 \\ 2 & 2 & 7,8 & 7 \cdot 5 \\ 1 & 2 & 4,4 & 4\end{array}$

the organism ceases to move after much fewer revolutions and between 1 and 10 sec. the revolutions vary directly with the duration of the rest period.

The recuperative power of these organisms seems to be extraordinarily rapid, for even after a period of rest of only 1 sec. an organism which was apparently incapable of movement is able to resume movement for some 15-20 sec.

In these observations flagellar movement started a fraction of a second before movement of the whole organism. At its fastest this organism made 1 revolution in 2 sec. This seems too slow to throw out mucoid substance. The flagella were ready to move as soon as they received the next stimulus. There was no question of the organism moving first and throwing out the flagella. The flagella moved first.

In the long filamentous forms it is common to find a filament moving with undulations of the body just like a serpent, but such a filament very frequently bends over into a close $U$-shape and moves forward with the bend of the $U$ in front without any undulations of the limbs, and with rope-like flagella in active movement streaming backwards from inside the bend, as reported also by Kingma Boltjes (1948). In a previous communication instances were given of 
movements which seemed impossible by simple undulations of the body (Fleming, 1949).

The movements of the organism may be so sluggish that it takes a coiled filament 30 sec. or more to make a single revolution round the central axis. It is inconceivable to us that this slow movement would be capable of throwing off mucoid threads, which, according to Pijper, constitute the so-called flagella. Moreover, the flagella, even in these slow-moving organisms, can easily be seen by phase contrast to be in very active motion. It is much more believable that the flagella are doing their best to move the organism, but by the physical conditions the movement is hampered and very slow.

Quite commonly flagellar movement can be seen in these slide cultures when the swollen organisms appear to be so hemmed in by their neighbours that they cannot move. The flagella here react to heat rays exactly as the flagella of the organisms which are capable of motion.

The movement of these active flagella attached to non-motile organisms was timed in the same way as the movement of motile organisms (Table 2). The

Table 2. The reaction to heat of flagella on stationary organisms

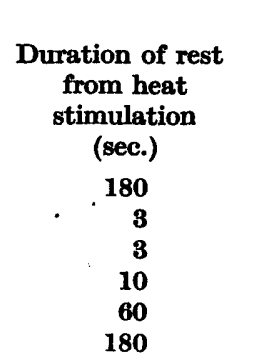

Latent period before
movement of flagella
recommenced after
heat stimulation
(sec.)
1
7
-8
About 1
About 1

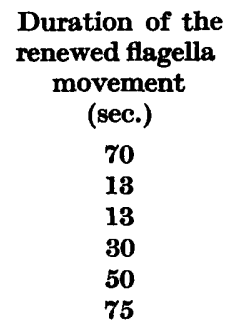

figures show that after a long interval of rest the latent period was much shorter and the length of time during which the flagella were active was much longer. After being stimulated many times by short periods of rest, the flagella appeared to become completely exhausted and ceased to respond to the heat stimulus. Clearly these active flagella, which can be stopped and started at will by exposure to heat rays, cannot be merely mucoid strands cast off by the active movement of the organism as Pijper maintains. There is no movement to cast them off. It might be held that before the organism became closed in by its neighbours so that it could not move, it had cast off these mucoid strands which still remained attached to the body. If that were so it would be strange if these inanimate strands were capable of responding to heat by going into active motion exactly as though attached to organisms which can move. In these the flagella stop and the organism stops when the heat filter is introduced again. Movement of the flagella and of the organism starts again when the heat filter is removed but the body never moves before the flagella.

It has not been possible to see the flagella by phase contrast where there was fluid between the agar and the cover-slip. In such conditions the motility of the organism was usually greater, but even where it was sluggish the flagella 


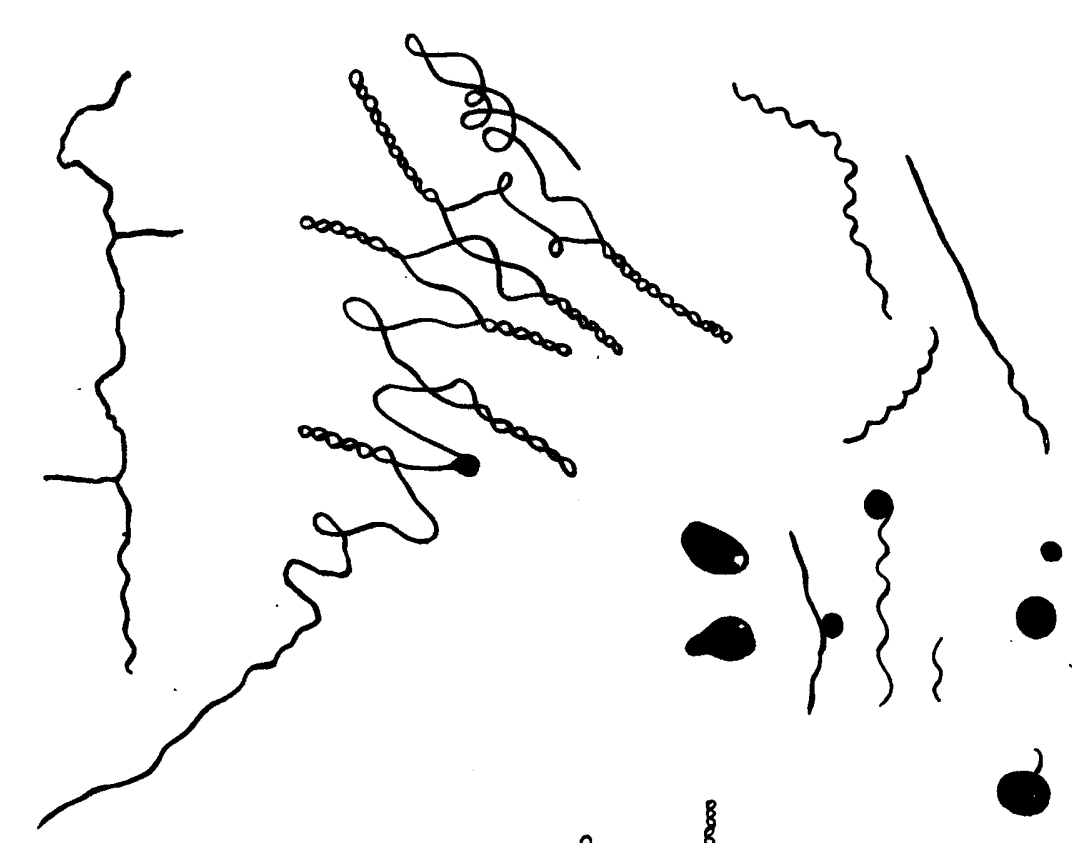

mperys

हु? 
could not be seen. Doubtless this is because, in these circumstances, the flagella, arising as they do from the whole surface of the organism, are projecting in all directions, when whereas the organism is lying between the agar and the cover-slip they can only be in one plane and so are more readily focused. The movement of the spherical forms is of some interest. We have shown that they possess flagella. The position of the vacuoles permits orientation of the sphere to be determined during the whole period of observation. Even a rolling movement of the body could be excluded; it seemed clear that motility was due to some outside influences and the most likely outside influences were the flagella.

These organisms were large-if movement were due to gyratory undulations of the body then one would expect to see such undulations, but usually the body showed nothing of the sort. The movements are frequently such that it would be impossible for them to take place unless we suppose they are caused by a pushing movement of something outside the body and if we reject Pijper's contention and accept the old idea that the flagella are actually the organs of motility, all the movements can be explained.

We have succeeded in preparing stained specimens in undisturbed slide cultures of $\boldsymbol{P}$. vulgaris by the method indicated earlier. The bacteria were arranged as seen in the living culture; the flagella were clearly visible-spread out where the bacteria were not closely opposed or in wavy 'ropes', where the bacteria were close to each other or where the coiled filaments of the same organisms were closely apposed.

\section{Organisms other than Proteus vulgaris}

We have discussed the changes in $\boldsymbol{P}$. vulgaris grown in penicillin. Very similar morphological changes can be observed with Salmonella typhi using the same penicillin concentrations. Swellings and protozoon-like forms are common, but more especially there is a predominance of long filamentous forms.

Bacterium coli showed the same changes, but to a lesser degree, with concentrations of $50-500 \mathrm{u} . / \mathrm{ml}$. penicillin in the agar slide cultures.

Vibrio cholerae, grown in concentrations of penicillin of 5-20 u. $/ \mathrm{ml}$., show large numbers of spiral organisms. It appeared as if the organisms grew up to the point at which separation should have taken place, but there the process stopped. Each element had the typical curved form, but instead of being separate curved vibrios they had the spirillum-like appearance. Frequently this was still more exaggerated and single filaments could be seen which had twisted themselves into extraordinary shapes (Fig. 3).

In stronger concentrations of penicillin and especially at $37^{\circ}$ all the organisms had grown into bulbous protozoon-like elements resembling those of $\boldsymbol{P}$. vulgaris but smaller. Our results with Vibrio cholerae largely confirm those of White (1950).

Pseudomonas pyocyanea was more resistant but in 5000-10,000 u./ml. of penicillin agar long threads and bulbous enlargements occurred. Sometimes branched forms were observed (Fig. 4). 
We wish to thank Mr Ben May of Mobile, Alabama, for a research grant to one of us and to Mr Mry and the American Optical Company for gifts of phase-contrast microscopes which enabled this work to be done, and Dr Cardew of the Photographic Department here for his help and advice.

\section{REFERENCES}

Ainiey Walker, E. W. \& Murray, W. (1904). The effect of certain dyes upon the cultural characters of Bacillus typhosus and other micro-organisms. Brit. med. J. ii, 16.

Dugum, J. P. (1948). The influence of cultural conditions on the morphology of bacterium aerogenes with reference to nuclear bodies and capsule size. J. Path. Bact. 60, 265.

Fleming, A. (1949). The Nature of the Bacterial Surface, p. 166. Edited by A. A. Miles and N. W. Pirie. Oxford: Blackwell's Scientific Publications Ltd.

Gardner, A. D. (1940). Morphological effects of penicillin on bacteria. Nature, Lond., 146, 837.

Kingma Boltues, T. Y. (1948). Function and arrangement of bacterial flagella. J. Path. Bact. 60, 275.

KunENEBERger-NobeL, E. (1949). Origin, development and significance of L-forms in bacterial cultures. J. gen. Microbiol. 3, 439.

Prjper, A. (1946). Shape and mobility of bacteria. J. Path. Bact. 58, 325.

Robinow, C. F. (1945). Addendum to The Bacterial Cell, ed. R. J. Dubos. Cambridge, Mass. : Harvard Univ. Press.

Spray, G. H. \& Lodge, B. M. (1943). The effects of resoreinol and of $m$-cresol on the growth of Bact. lactis aerogenes. Trans. Faraday Soc. 39, 424.

WEBB, M. (1948). The influence of magnesium on cell division. J. gen. Microbiol. 2, 275.

White, P. B. (1950). A note on the globular forms of V. cholerae. J. gen. Microbiol. 4, 36 .

\section{EXPLANATION OF PLATES}

\section{Plate 1}

Figs. 1-6. The development of abnormal forms in contact with penicillin. Serial photographs by phase contrast. $(\times 860$.)

Fig. 1. A young culture $1 \frac{1}{2} \mathrm{hr}$. after seeding on to penicillin agar $5 \mathrm{u} . / \mathrm{ml}$. Originally all the organisms were the same size; growth and division of the two lower organisms.

Fig. 2. After $4 \mathrm{hr}$. Long threads with well-marked nuclear dots.

Fig. 3. After $4 \frac{1}{2} \mathrm{hr}$. Swellings appear on the threads and a loop forms at the tip of the righthand bundle.

Fig. 4. After $5 \mathrm{hr}$. Part of the right-hand bundle streams down to form a rotating spiral.

Fig. 5. After $6 \frac{1}{\mathrm{~d}} \mathrm{hr}$. Swellings continue to develop. The first-formed spiral has ceased to rotate, and a new one has formed from the upper loop of the left-hand group.

Fig. 6. After $19 \mathrm{hr}$. Vacuolation well marked. Fragmentation of threads.

Plate 2

Fig. 7. Bacilli in the early stages of penicillin-induced change, stained for nuclei. ( $\times 2150$.)

Figs. 8, 9. More advanced stages, showing condensation of nuclear material. $(\times 4300$.

Figs. 10-12. Larger forms showing condensed nuclear material. ( $\times 2150$.)

Fig. 13. Large swellings. Diffuse nuclear material.

Fig. 14. Large swellings. Central condensation of nuclear material.

Fig. 15. Large swellings. Reticular pattern of nuclear material.

Fig. 16. Very large swelling with central reticular nucleus. 
Journal of General Microbiology, Vol. 4, No. 2
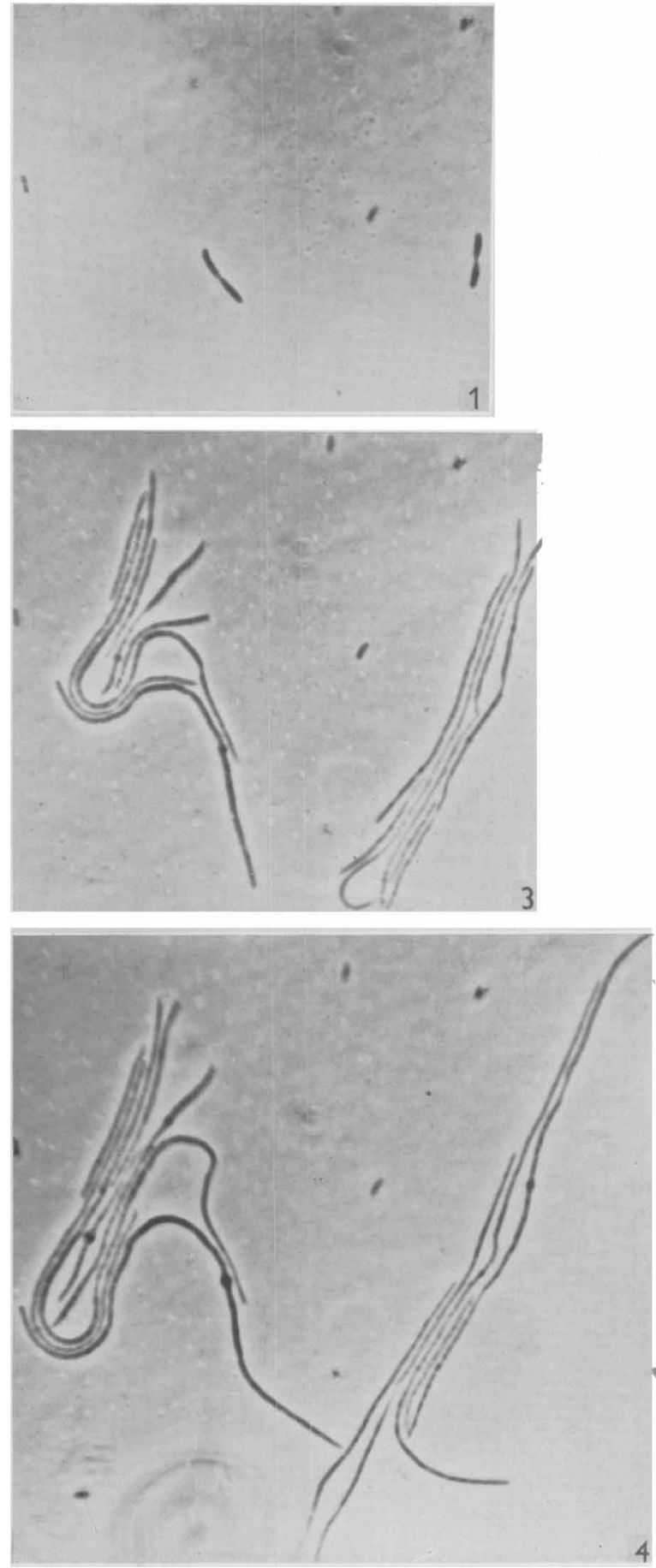

Figs. 1-6
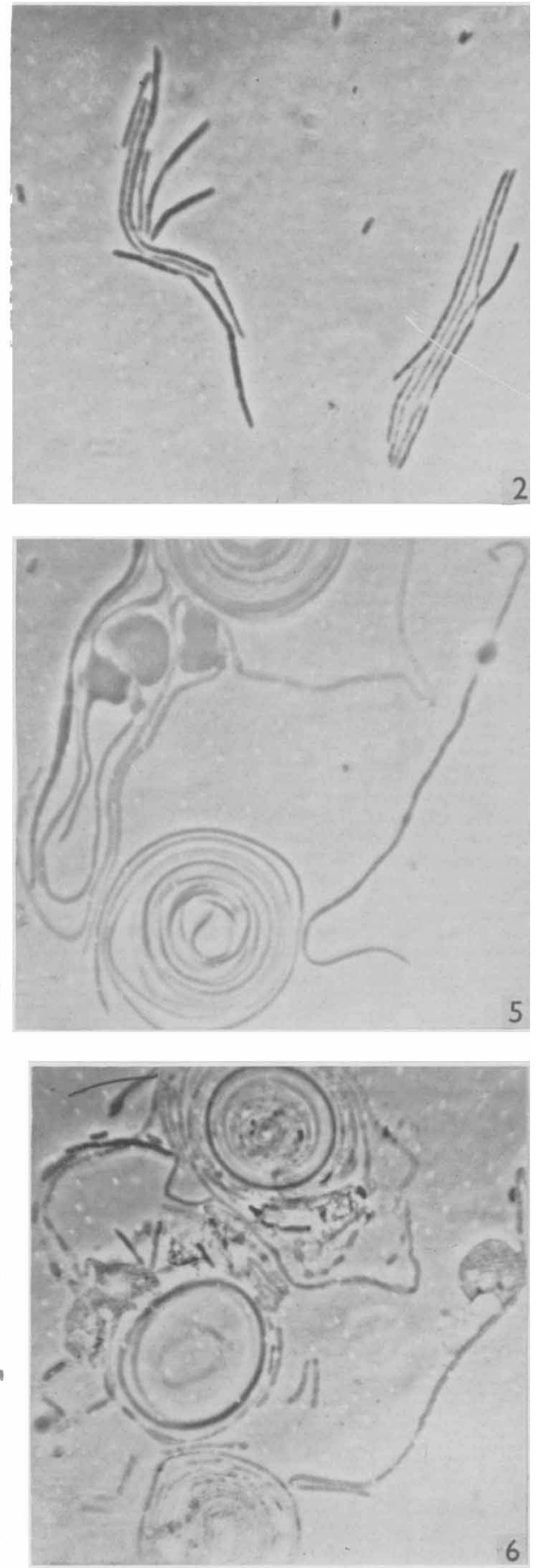

6

A. Fleming, A. Voureka, I. R. H. Kramer \& W. H. Hughes-Penicillin-induced changes in P. vulgaris. Plate 1 
Journal of General Microbiology, Vol. 4, No. 2

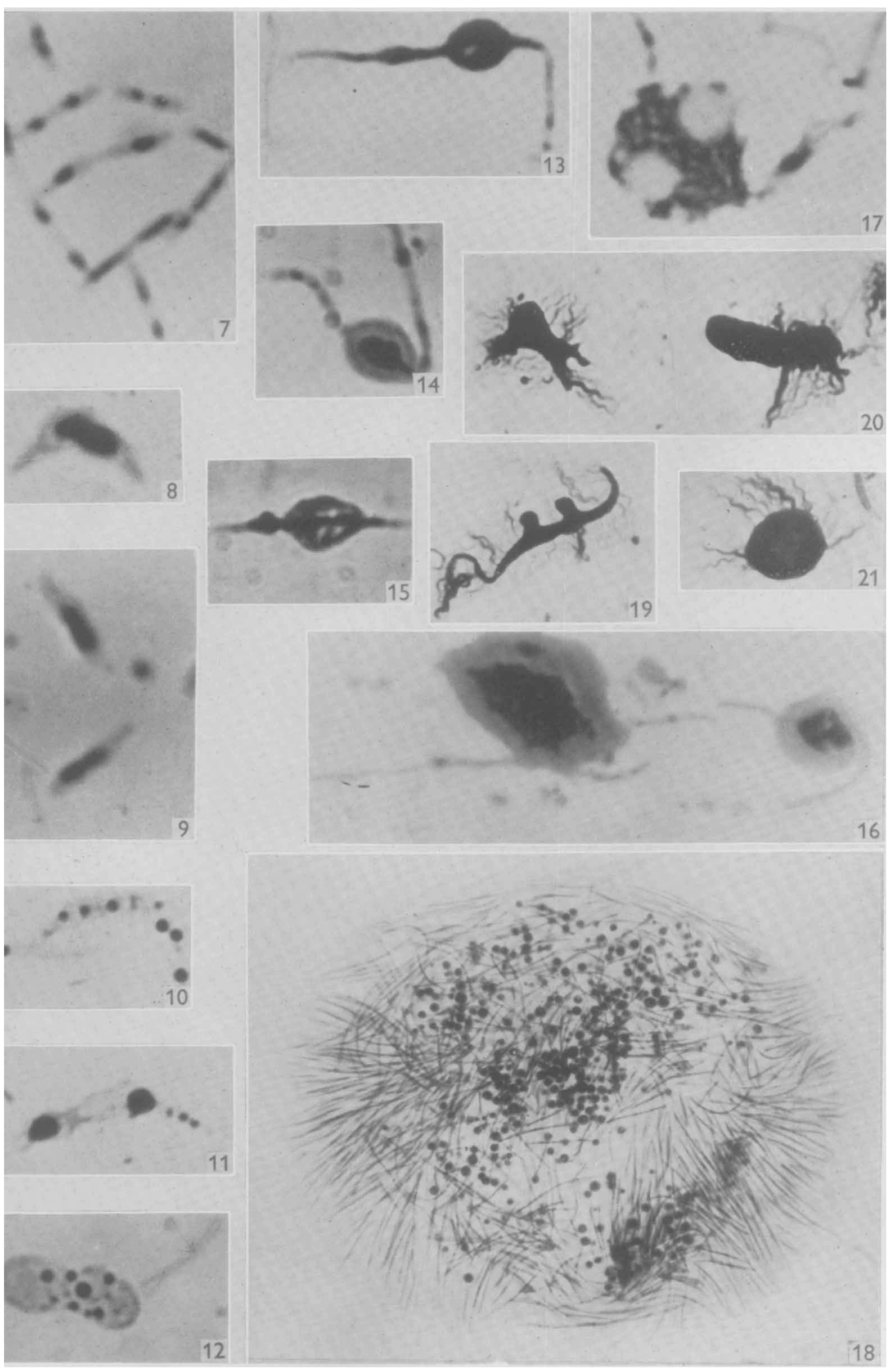


Journal of General Microbiology, Vol. 4, No. 2

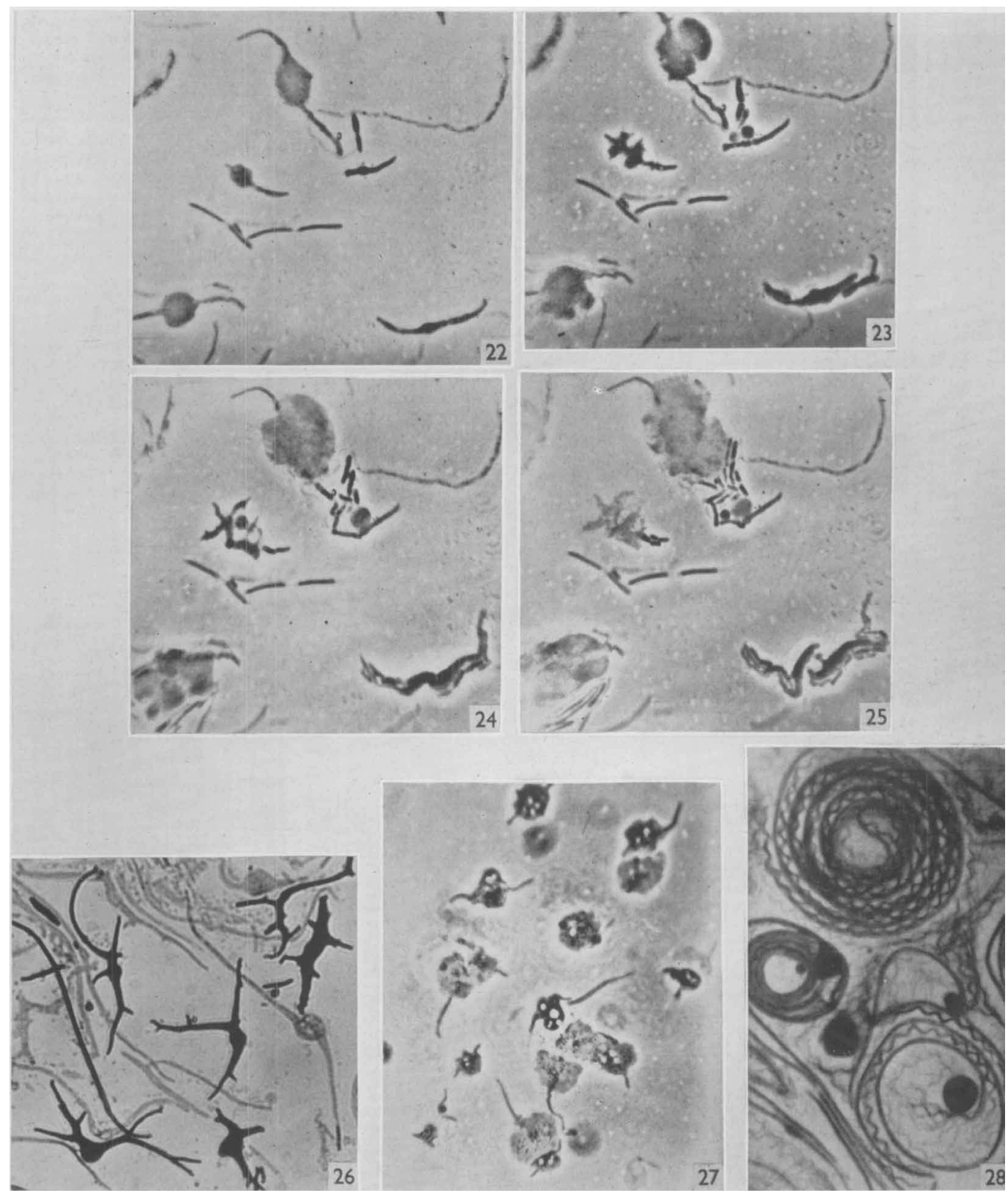

Figs. 22-28

A. Fleming, A. Voureka, I. R. H. Kramer \& W. H. Hughes-Penicilin-indechd changes in P. vulgaris. Piate 3 


\section{Penicillin-induced changes in $\mathbf{P}$. vulgaris}

Fig. 17. Vacuolated swelling, reticular nucleus displaced by vacuoles.

Fig. 18. 'Pool' with threads resting on the edge and spherical forms in the deeper layer of fluid at the centre. ( $\times 250$.)

Figs. 19-21. Flagella of abnormal forms.

\section{Prate 3}

Figs. 22-25. The degenerate cover-slip culture shown in Pl. 1, fig. 6, was transferred to penicillinase-agar to allow recovery to take place. Serial photographs by phase contrast. $(\times 720$.

Fig. 22. Immediately on transference to penicillinase-agar.

Fig. 23. After $1 \mathrm{hr}$. Swellings enlarge, the more normal forms begin to divide. Tails of swellings divide.

Fig. 24. Swellings begin to disintegrate.

Fig. 25. Tails and surviving cells continue to divide to give bacilli of normal size. A number of organisms in the field show no sign of recovery though not necessarily as abnormal in appearance as those that resume growth.

Fig. 26. Branched forms and ghosts, 20 u. penicillin/ml. $(\times 720$.

Fig. 27. Field of cells all affected by a high concentration of penicillin, $50 \mathrm{u} . / \mathrm{ml}$,

Fig. 28. Stained spirals showing flagellá ropes.

(Received 21 October 1949) 